\title{
Linking Spatial Processes to Life-History Evolution of Insect Parasitoids
}

\author{
T. S. Hoffmeister, ${ }^{1,3, *}$ B. D. Roitberg, ${ }^{2, \dagger}$ and L. E. M. Vet ${ }^{3,4, \star}$
}

1. Institute of Ecology and Evolutionary Biology, University of Bremen, D-28359 Bremen, Germany;

2. Department of Biological Sciences, Simon Fraser University, 8888 University Boulevard, Burnaby, British Columbia V5A 1S6, Canada;

3. Department of Multitrophic Interactions, Netherlands Institute of Ecology (NIOO-KNAW), P.O. Box 40, 6666 ZG Heteren, The Netherlands;

4. Laboratory of Entomology, Wageningen University,

Binnenhaven 7, 6709 PD Wageningen, The Netherlands

Submitted January 5, 2005; Accepted April 23, 2005;

Electronically published July 11, 2005

ABSTRACT: Understanding the evolutionary transition from solitary to group living in animals is a profound challenge to evolutionary ecologists. A special case is found in insect parasitoids, where a tolerant gregarious larval lifestyle evolved from an intolerant solitary ancestor. The conditions for this transition are generally considered to be very stringent. Recent studies have aimed to identify conditions that facilitate the spread of a gregarious mutant. However, until now, ecological factors have not been included. Host distributions and life-history trade-offs affect the distribution of parasitoids in space and thus should determine the evolution of gregariousness. We add to current theory by using deterministic models to analyze the role of these ecological factors in the evolution of gregariousness. Our results show that gregariousness is facilitated through inversely density-dependent patch exploitation. In contrast, host density dependence in parasitoid distribution and patch exploitation impedes gregariousness. Numerical solutions show that an aggressive gregarious form can more easily invade a solitary population than can a tolerant form. Solitary forms can more easily invade a gregarious, tolerant population than vice versa. We discuss our results in light of exploitation of multitrophic chemical cues by searching parasitoids and aggregative and defensive behavior in herbivorous hosts.

Keywords: clutch size, density dependence, competition, foraging behavior, spatial heterogeneity, multitrophic interactions.

\footnotetext{
* Corresponding author; e-mail: hoffmeister@uni-bremen.de.

† E-mail: bernard_roitberg@sfu.ca.

₹ E-mail: l.vet@nioo.knaw.nl.
}

Am. Nat. 2005. Vol. 166, pp. E62-E74. (C) 2005 by The University of Chicago. 0003-0147/2005/16603-40830\$15.00. All rights reserved.
The transition from solitary lifestyle to group living in animals has intrigued behavioral ecologists (Krebs and Davies 1993; Prokopy and Roitberg 2001). Group living evolves when the benefits outweigh costs (Wertheim et al. 2005) like increased competition, transmission of pathogens and parasites, cannibalism, or cuckoldry (Krebs and Davies 1993). Benefits of group living may come from increased vigilance (e.g., Bertram 1980) or dilution (e.g., Perez-Contreras et al. 2003) and confusion effects against predators (e.g., Neill and Cullen 1974) or Allee effects (Rohlfs and Hoffmeister 2003). A special case is the evolution of gregarious development in parasitoid wasps. Parasitoids lay one or more eggs in or on other insects, and their larvae feed on and kill the host. Gregarious development resembles the nursery of birds where few of the above-mentioned benefits of group living may play a role. The common benefit is that locally abundant resources can be optimally allocated to a group of offspring. By being gregarious, a parasitoid can attack larger hosts or increase its Lack clutch size through smaller progeny.

A seminal article by Godfray (1987) addressed the evolutionary transition from solitary toward gregarious development. Solitary wasp larvae are killers; only a single parasitoid larva can successfully develop on/in a given host. When more eggs are deposited, elimination takes place (Godfray 1994) even if the host provides enough food for the development of several larvae. In gregarious species, several parasitoid larvae can develop together on a single host, and siblicide is generally absent. Godfray (1987) assumed that in hosts inhabited by both a solitary and a gregarious larva, the gregarious larva is always killed in the highly asymmetric competition. Hence, one of the major conclusions of Godfray's genetic model was that it was difficult for the tolerant gregarious strategy to evolve once the population comprises nontolerant solitary individuals (see also Harvey and Partridge 1987). Solitary development represents the ancestral life-history state (Mayhew 1998a; Ode and Rosenheim 1998), but the transition to gregariousness has occurred on many occasions (Mayhew 
1998a). It thus seems that the evolutionary transition is less problematic than theory suggests. Considerable effort has been put into identifying factors that facilitate the spread of a tolerant allele. There is now theoretical and empirical evidence that costs of fighting, female-biased sex ratios, size asymmetries among sibs, single-sex broods, and, especially, reduced larval mobility all facilitate the spread of a tolerant allele (Rosenheim 1993; Rosenheim and Hongkham 1996; Mayhew 1998a, 1998b; Mayhew and Hardy 1998; Mayhew et al. 1998; Ode and Rosenheim 1998; Mayhew and Van Alphen 1999; Boivin and van Baaren 2000; Pexton and Mayhew 2001, 2004; Pexton et al. 2003). The evolution of gregariousness critically depends on the degree of interactions between larvae; immobility of larvae facilitates gregariousness, while superparasitism, that is, the parasitization by more than one conspecific female, operates against the spread of a tolerant allele (Godfray 1987; Rosenheim 1993; Pexton et al. 2003).

The above-mentioned models use a genetic approach, mainly focusing on the interaction between the offspring of a single female. In this article, we envisage an additional approach, where we focus on ecological conditions that influence the strength of interaction between the offspring of different (tolerant and nontolerant) females. We expect behavioral responses to spatial heterogeneity (e.g., searching in a host density-[in]dependent manner) to affect the probability of interaction between solitary and gregarious parasitoids that are simultaneously exploiting a host population. Such an ecological approach has not previously been considered.

Similarly, differences in trade-offs between solitary and gregarious life-history strategies, which we believe are essential for understanding the ecology and evolution of these different strategies, have scarcely been considered (but see Pexton and Mayhew 2002). Solitary parasitoids produce only one offspring per host irrespective of the amount of resources a host provides. Gregarious parasitoids, by contrast, can produce more offspring per host, even without severe scramble-type competition. This especially holds for hemolymph feeding parasitoids that allow their hosts to develop further after parasitization (Harvey et al. 2000; Harvey and Strand 2002). While a solitary larva leaves a considerable portion of the host unused, gregarious development can make full use of the host resource.

General life-history theory (Roff 1992; Stearns 1992) predicts a trade-off between fecundity and longevity (a cost of reproduction; e.g., Sevenster et al. 1998; Ellers et al. 2000b). Because of this trade-off, gregarious parasitoids that use more eggs per host may have shorter life spans than solitary parasitoids (e.g., Laing and Levin 1982; Nealis 1990; Geervliet 1997; Pexton and Mayhew 2002), which translates to fewer opportunities for encountering hosts.
Thus, there is a need to consider evolution of gregarious parasitoid life history in a spatial context.

Here we use deterministic models to detect conditions that permit the evolution of the gregarious life history in solitary parasitoid populations. Where analytical solutions are unfeasible, we use numerical solutions.

\section{The Model}

In contrast to Pexton and Mayhew (2001), we assume that a double mutation has changed both behavior and clutch size at once. In a population of solitary individuals, we introduce such a mutant. Its new behavior can be either tolerance or larval immobility. Empirical data for different parasitoid species show that both are possible (Pexton and Mayhew 2001), and we will deal with each in turn.

In the case of larval tolerance, competition between solitary and gregarious forms is highly asymmetric. Hence, the fitness of a gregarious individual depends on the frequency of interactions with solitary individuals (but not the other way around). We therefore apply a game theoretical approach; that is, we search for conditions under which a gregarious mutant is able to invade a population of solitary parasitoids. We are not concerned about the genetic system involved. Rather we assume that strategies replicate asexually to keep the ecological interactions we are interested in tractable. We realize that haplodiploid inheritance complicates the spread of a gregarious allele considerably as a result of the fact that some of the offspring will be of mixed phenotype and gregarious offspring are lost through within-brood interactions. This problem has been dealt with earlier, and we focus entirely on the interactions between broods. Inclusion of mixed phenotype broods would make the conditions for the spread of gregariousness much more stringent than stated in our results. Nevertheless, the results of our models elucidate situations that in general will facilitate or hamper the evolution of gregariousness.

In our approach, we link interactions between solitary and gregarious parasitoids to their foraging behavior in patchy habitats. We calculate the marginal clutch size, that is, a mutant's clutch size that leads to the same number of surviving offspring as an average solitary parasitoid produces. Any higher clutch size of the gregarious parasitoid would then allow the gregarious strategy to spread in a population of solitary parasitoids. Clearly, the marginal clutch size will depend on the reproductive success a solitary parasitoid can gain in the habitat and the chance that the gregarious mutant will be the sole exploiter of a host wherein her offspring escape fatal competition with solitary offspring.

Below, we will describe our models verbally and present 

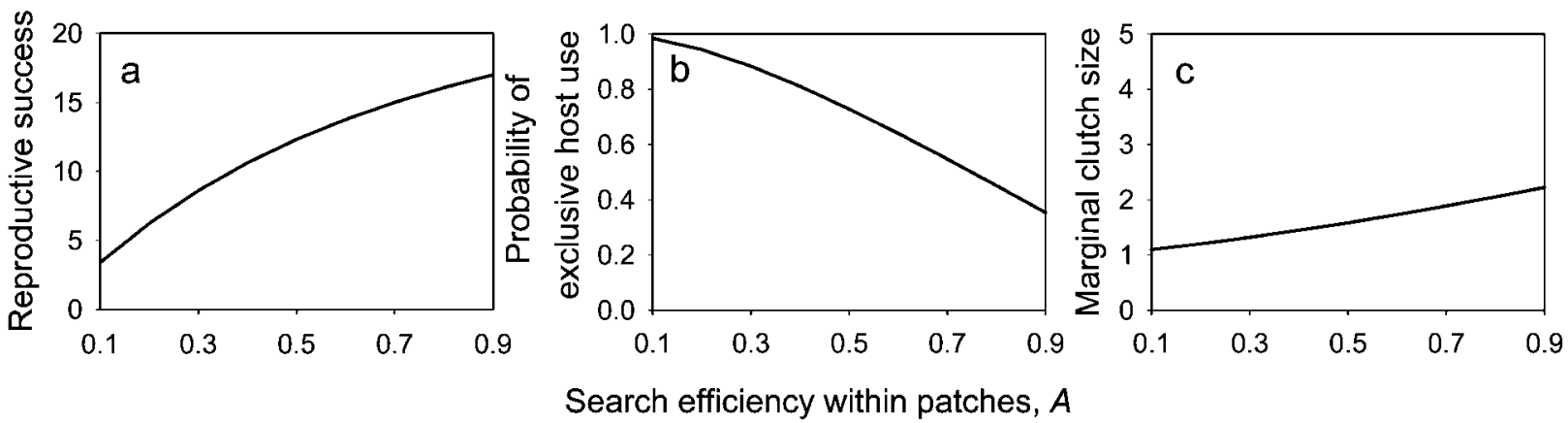

Figure 1: Effect of the proportion of hosts each parasitoid parasitized within a patch on $(a)$ reproductive success of solitary parasitoids (measured as average number of surviving offspring a solitary parasitoid can obtain in a patch with 50 hosts), (b) probability of exclusive host use by a gregarious parasitoid, and $(c)$ the marginal clutch size of the gregarious mutant, that is, the minimal clutch size of a gregarious parasitoid needed to invade a population of solitary parasitoids. All patches have equal host densities.

the results in figures. The underlying mathematical equations are presented in the appendix.

We envision a habitat that contains hosts distributed between patches of either the same or different host density. A population of solitary parasitoids plus a single gregarious mutant are distributed randomly across these patches. In some scenarios, we introduce a bias wherein some patches differ in their attractiveness to parasitoids. Within patches, we assume random search for hosts that are accepted if unparasitized or conspecifically parasitized (i.e., no self-superparasitism occurs). This is an important assumption. The avoidance of self-superparasitism is common in parasitoids (van Dijken et al. 1992) and can be a key to an efficient use of the egg supply (but see Visser 1993 for conditions for adaptive self-superparasitism). However, acceptance of conspecifically parasitized hosts (a conditional strategy in many parasitoids; van Alphen and Visser 1990) is essential to allow for the interaction of solitary and gregarious parasitoids within hosts in our model. In nature, such conspecific superparasitism depends on host availability; thus, the general acceptance of a parasitized host is a worst case, most stringent scenario. In each scenario, we start with the measurement of the expected reproductive success of a solitary parasitoid, which depends on the distribution of all solitary parasitoids in the habitat. Note that the interaction with the tolerant gregarious parasitoid is irrelevant to our focal solitary parasitoid because tolerant gregarious larvae do not harm solitary larvae. The probability that a given host is exclusively exploited by the gregarious mutant, our second measurement, depends on the density and exploitation level of the solitary parasitoids foraging within the same patch.

Some gregarious offspring will be killed as a result of interactions with offspring from solitary parasitoids.
Hence, gregarious parasitoids must provide each host they attack with a marginal clutch size to compensate for the loss in competition; this is our third measurement, the marginal clutch size.

\section{Scenario 1: Some Are Proficient: Effects of Parasitoid Exploitation Level within Patches}

We first investigate the effects of increasing interaction between parasitoids by varying the percentage of hosts each parasitoid attacks within a patch. With increasing exploitation level, the reproductive success of a solitary parasitoid increases (fig. 1a). However, reproductive success does not increase linearly with the exploitation level because of increasing superparasitism leading to the elimination of supernumerary larvae. At the same time, the probability of exclusive host use by the gregarious parasitoid decreases (fig. $1 b$ ). While solitary parasitoids accrue an equal share of reproductive success from superparasitized hosts, the tolerant gregarious mutant always loses the interaction with the fighting solitary. To compensate for these losses, gregarious parasitoids must increase their clutch size at increased exploitation levels (fig. 1c). In conclusion, the spread of the tolerant strategy is most likely to occur under low exploitation levels. As was found in Godfray's (1987) population genetics model, increased probability of superparasitism makes the conditions under which gregariousness can evolve more stringent.

\section{Scenario 2: Going for Crowds: Effects of Host Density-Dependent Distribution of Parasitoids across Patches}

In scenario 1, all patches in the habitat are of equal host density. In reality, patches vary with respect to host density. 
Empirical evidence (e.g., Godfray 1994) suggests that many parasitoid species search longer in higher-density patches. Thus, the proportion of hosts that are attacked by a parasitoid can remain constant across host densities. This is assumed here; thus, the probability of exclusive host use by a gregarious mutant remains constant under such density-independent exploitation, and the spread of the mutant tolerant strategy does not vary with host density alone.

Yet, patches with different host densities often differ in their apparency to parasitoids. Patch and host location in parasitoids are often governed by chemicals that bear information on the presence of hosts, so-called semio- or infochemicals (Dicke and Sabelis 1988). For example, herbivores can induce the production of specific volatile plant compounds that are highly attractive to their parasitoids (Vet and Dicke 1992). Volatile production by host plants (see Geervliet et al. 1998) or by the host insects themselves (Vinson 1984) can be host density dependent. We therefore explore a range of values from density independence with equal probabilities of parasitoids entering patches of different host density to full proportional density dependence where, on average, parasitoids enter patches at a constant ratio of parasitoids to hosts across all patches in the habitat. Note that still, the proportion of hosts that each parasitoid attacks within a patch remains constant across all host densities. For simplicity, we assume that the number of parasitoids that reach a patch increases linearly with host density (while in nature, threshold-based responses to volatile infochemicals may occur).

When higher-host-density patches attract more parasitoids, competition among solitary parasitoids increases in those patches. In spite of the latter, figure $2 a$ shows that the average reproductive success in the habitat increases slightly with increasing density-dependent parasitoid distribution. Because of the increased competition in highhost-density patches and the fact that the gregarious mutant most likely will also preferably enter the high-hostdensity patches, the probability of the gregarious mutant to be the sole exploiter of a given hosts decreases (fig. $2 b$ ). Therefore, positively density-dependent parasitoid distribution across patches hampers gregarious evolution. Consequently, a higher marginal clutch size is required to allow the evolution of gregariousness (fig. 2c). Figure 3 shows the marginal clutch size as a function of density-dependent parasitoid distribution and host density per patch. It illustrates that density dependence leads to a severe increase in the marginal clutch size of the gregarious mutant exactly in those high-host-density patches at the far end of figure 3 that most parasitoids enter. On the other hand, densitydependent parasitoid distribution also creates partial refuges in low-density patches on the very left side of figure 3 that attract few parasitoids and thus relax competition. In conclusion, positive density-dependent parasitoid distribution across patches makes it less likely that a gregarious strategy can spread in a population of solitary wasps.

\section{Scenario 3: Dealing with the Crowds: Effects of Density-Dependent Host Exploitation within Patches}

Parasitoid behavior within patches can, however, also be density dependent. All forms of within-patch density dependence have been described for host-parasitoid systems, from inverse density dependence to positive density dependence (Lessells 1985; Stiling 1987; Shiojiri and Takabayashi 2003). In this scenario, we add effects of densitydependent patch exploitation, keeping density dependence

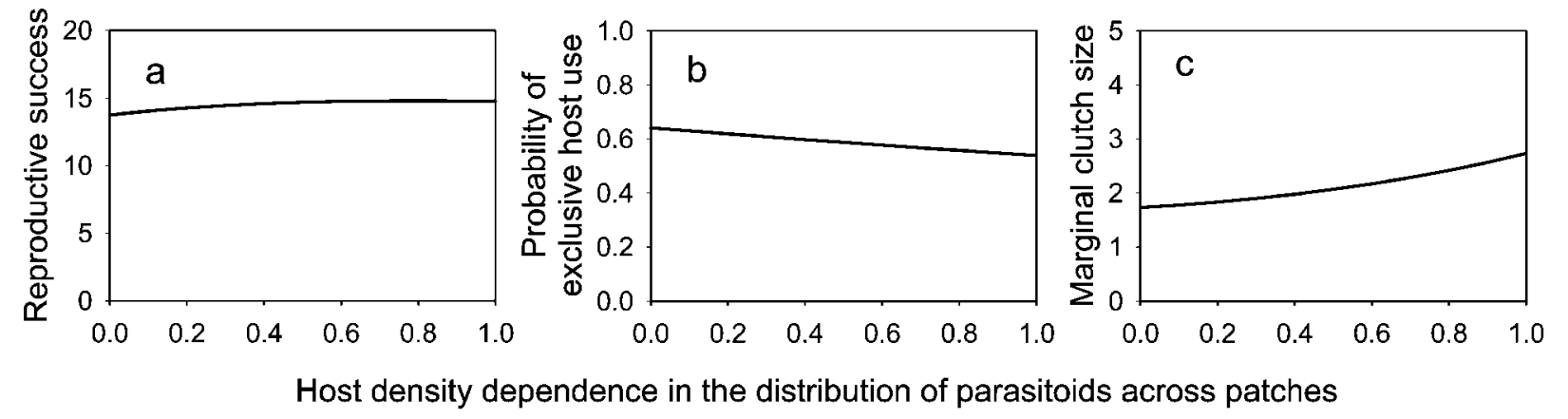

Figure 2: Effect of host density dependence in the distribution of parasitoids across patches on (a) average reproductive success of solitary parasitoids, (b) average probability of exclusive host use by a gregarious parasitoid, and (c) the average marginal clutch size of the gregarious mutant, that is, the minimal clutch size of a gregarious parasitoid needed to invade a population of solitary parasitoids (for calculations of average values, see the appendix); $x$ values represent no host density dependence at $x=0$ to full proportional host density dependence at $x=1$, where the distribution of parasitoids matches that of hosts across patches. 


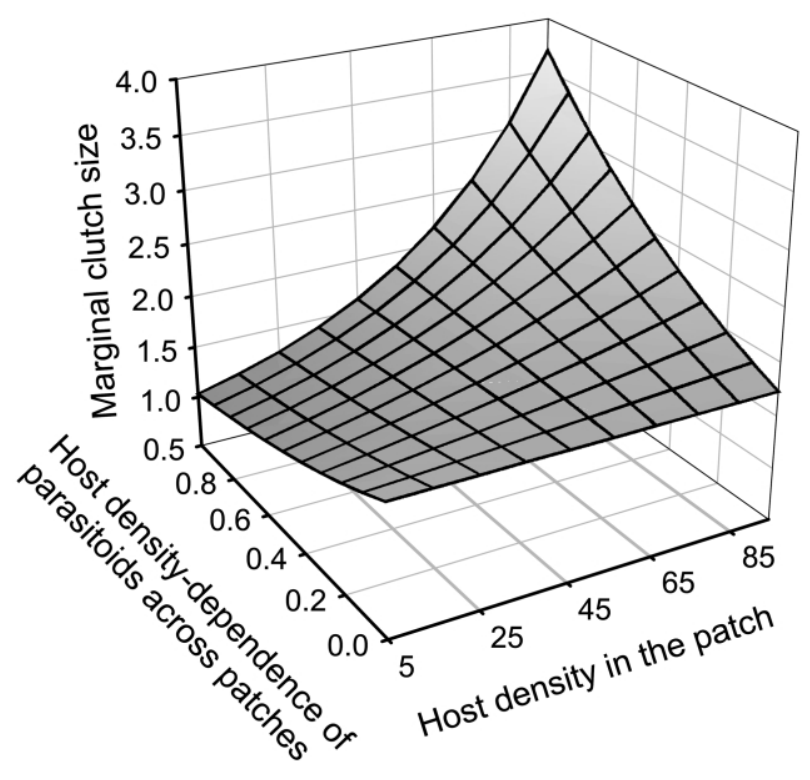

Figure 3: Effect of host density dependence in the distribution of parasitoids across patches and host density in a patch on the marginal clutch size of the gregarious mutant, that is, the minimal clutch size of a gregarious parasitoid needed to invade a population of solitary parasitoids. See figure 2 for details.

in parasitoid distribution across patches (previous scenario) fixed at proportional density dependence, that is, the right-hand-side values in figure 2 . With densityindependent patch exploitation, each parasitoid that visits a patch parasitizes $60 \%$ of the hosts in that patch. With full inverse density-dependent patch exploitation, each wasp attacks $95 \%$ of hosts in the lowest-host-density patches and $25 \%$ in the highest-host-density patches. In turn, with full positive density dependence, each wasp attacks $25 \%$ of hosts in the lowest-density patch and $95 \%$ in the highest-host-density patches.

We first focus on inverse density-dependent exploitation (negative $x$ values in fig. 4). The lower the proportion of hosts that is parasitized by each wasp in high-host-density patches, the lower is its reproductive success (fig. 4a). At the same time, the probability for the gregarious mutant to exploit hosts by herself increases (fig. $4 b$ ); thus, lower clutch sizes are sufficient for a gregarious mutant to invade the solitary parasitoid population (fig. $4 c$ ).

Next, positive density-dependent patch exploitation is depicted on the right-hand side of the graphs of figure 4 (with positive $x$ values). This situation is detrimental to the success of the gregarious mutant. An increased clutch size is necessary (fig. $4 c$ ) to allow its spread. This is due to the increased exploitation rate of attractive high-density patches that increases the reproductive success of solitary parasitoids (fig. $4 a$ ) and decreases the probability that the gregarious mutant exploits a host by herself (fig. $4 b$ ). In conclusion, only when high-host-density patches are relatively less exploited is the spread of the gregarious strategy facilitated.

\section{Scenario 4: The Seamy Side of Life (-History Trade- offs): Effects of Decreased Patch Location Probabilities in Gregarious Parasitoids}

Here, we investigate the effects of the life-history (i.e., fecundity-longevity) trade-off. This trade-off imposes a lower longevity on our gregarious mutant parasitoid to pay for her higher fecundity. Living less long translates into a lower chance of finding patches per lifetime, or, if we keep lifetime constant in our model, it translates into a lower chance to find patches per unit search time. In figure 6, this is expressed as the ratio of the probability of a gregarious and a solitary parasitoid locating a patch. Because a solitary offspring is unaffected by the presence or absence of a tolerant gregarious competitor, the reproductive success of solitary parasitoids and the probability of a gregarious mutant to monopolize a host once she has found a patch remain unchanged across the range of differential patch location probabilities. The only value that varies is the marginal clutch size (fig. 5). On the left-hand side of the graph, a gregarious mutant must compensate for lower patch location probabilities. On the right-hand side of the figure, the clutch size reflects only the compensation of losses in competitive interactions with solitary larvae. In conclusion, the life-history trade-off between fecundity and longevity combined with the need of higher fecundities in gregarious wasps makes it more difficult for gregarious development to spread.

\section{Tolerance versus Immobility}

Up to here we have assumed that the mutant gregarious wasp produces tolerant larval offspring that will always lose in the contest with fighting solitary larvae. There is, however, empirical and theoretical evidence (Boivin and van Baaren 2000; Pexton et al. 2003; Pexton and Mayhew 2004) that larval immobility can greatly facilitate the evolution of gregarious development. The most obvious difference between tolerant and immobile larvae is their effect on the reproductive success of the solitary parasitoids. Immobile mutant larvae do not kill each other because they do not encounter each other. If, however, a mobile solitary larva approaches them, they can attack and fight. Assuming an equal chance of survival of a gregarious and solitary larva in a contest, we used numerical solutions to compare the marginal clutch size aggressive immobile and tolerant gregarious mutants would need to invade a population of solitary wasps (see appendix for details). As long as solitary 

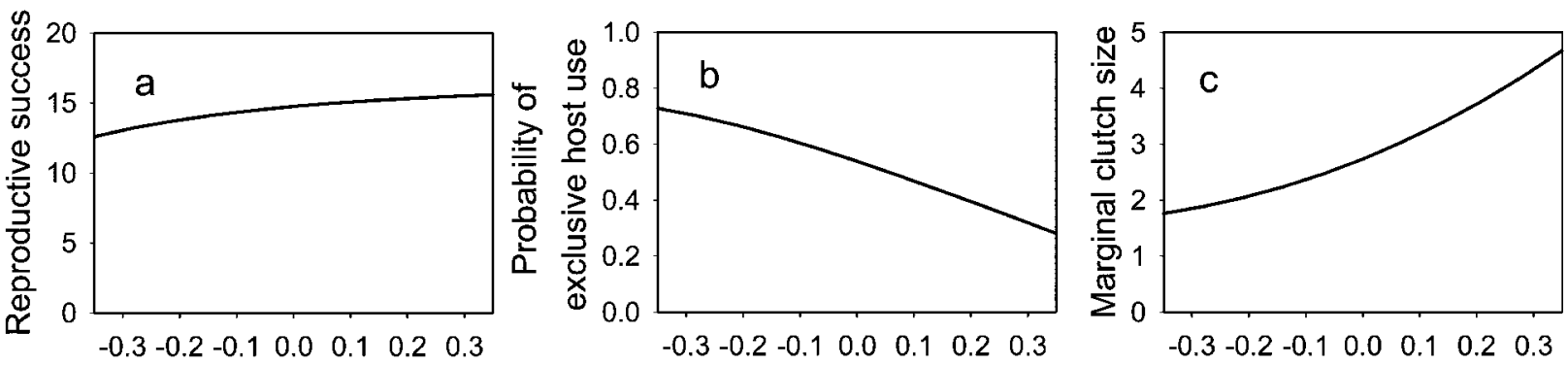

Host-density dependence in patch exploitation

Figure 4: Effect of host density-dependent patch exploitation, that is, proportion of hosts attacked per wasp, on $(a)$ average reproductive success of solitary parasitoids, $(b)$ average probability of exclusive host use by a gregarious parasitoid, and $(c)$ the average marginal clutch size of the gregarious mutant, that is, the minimal clutch size of a gregarious parasitoid needed to invade a population of solitary parasitoids (for calculations of average values, see appendix). $x=0$ represents host density-independent patch exploitation; increasingly negative $x$ values represent increasingly inverse host density-dependent patch exploitation, and increasingly positive $x$ values represent increasingly positive host density-dependent patch exploitation. Note that parasitoids are distributed in proportion to the host density across patches as in figure 2 .

and immobile aggressive gregarious wasps do not differ with respect to other features of their biology, any clutch size $>1$ should lead to successful invasion by the mutant. Consequently, the most interesting scenario to consider is scenario 4 , where gregarious wasps pay a price for their high fecundity. Congruent with the findings of Pexton et al.'s (2003) population genetics models, gregarious mutants with aggressive larvae need lower marginal clutch sizes than gregarious mutants with tolerant larvae (fig. 6).

\section{From Peace to War: Exploring the Transition from Tolerance to Fighting}

How stringent are the conditions for the spread of tolerance in our ecological scenarios? To answer this question, we explore conditions under which a solitary fighting strategy can invade a population of tolerant gregarious individuals. Here we explicate this situation using again our fourth scenario. As can be seen in figure 7, once gregarious wasps have successfully invaded a solitary population and have gone to fixation, it is not easy for a solitary mutant to reinvade. Because gregarious offspring do not kill each other within superparasitized hosts, gregariousness is selffacilitating once most nonsib larval encounters take place between gregarious offspring. Note, however, that we assume here that such offspring does not suffer from competition through, for example, reduced size that would translate into reduced fecundity and dispersal ability. Under such conditions, three regions in figure 7 can be distinguished: in the white region, gregarious mutants can invade a population of solitary wasps and are stable against reinvasion of solitary mutants. In the black region, gregarious mutants cannot invade a solitary population.
However, given a gregarious population, a solitary mutant would be equally incapable of invading the gregarious population. Finally, in the gray region, solitary mutants can successfully invade and are stable against reinvasion by gregarious individuals.

\section{Conclusions}

The results of our scenarios suggest that the evolution of gregariousness is facilitated whenever some mechanism leads to a decrease in interaction between a gregarious

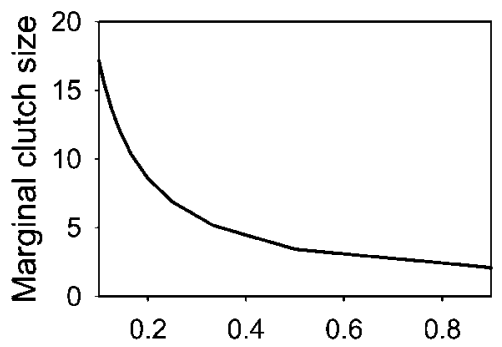

Ratio of patch location probabilities $(\lambda \mathrm{g} / \lambda \mathrm{s})$

Figure 5: Effect of lower patch location probability in gregarious parasitoids on the marginal clutch size of the gregarious mutant, that is, the minimal clutch size of a gregarious parasitoid needed to invade a population of solitary parasitoids. All patches have equal host densities. The ratio of patch location probability is expressed as the probability of a gregarious parasitoid to locate a given patch divided by the probability of a solitary parasitoid to locate a patch. Low $x$ values represent low patch location ability in gregarious parasitoids due to the life-history trade-off between longevity and fecundity; high $x$ values represent similar patch location ability in gregarious and solitary parasitoids. 


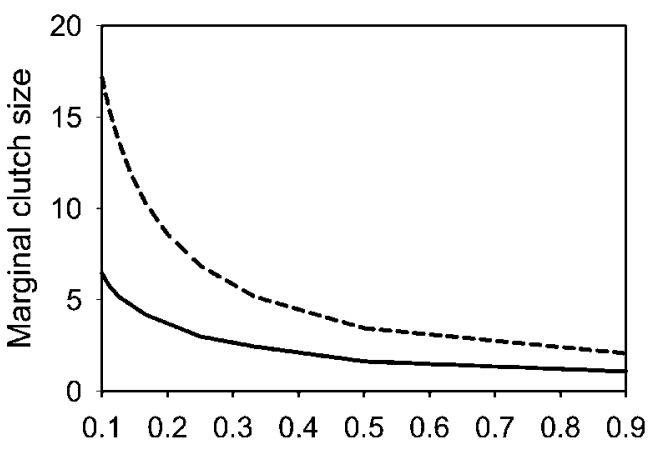

Ratio of patch location probabilities $(\lambda g / \lambda s)$

Figure 6: Comparison of the marginal clutch sizes for successful invasion of a solitary population by gregarious mutants when gregarious larvae are tolerant (dashed line) or aggressive (solid line). The ratio of patch location probability is expressed as the probability of a gregarious parasitoid to locate a given patch divided by the probability of a solitary parasitoid to locate a patch. Low $x$ values represent low patch location ability in gregarious parasitoids because of the life-history trade-off between longevity and fecundity; high $x$ values represent similar patch location ability in gregarious and solitary parasitoids.

parasitoid and its competitively superior solitary conspecifics. We showed that these mechanisms can derive from parasitoid behavioral response to spatial heterogeneity in their host distribution. While increased patch exploitation levels (fig. 1) and positive density dependence impede the evolution of gregariousness (figs. 2, 3), inverse densitydependent patch exploitation facilitates it (fig. 4). Additionally, when a higher fecundity of gregarious parasitoids trades off strongly against lifetime opportunities of locating patches, gregarious parasitoids must lay very large clutches (fig. 5). Finally, a comparison between aggressive and tolerant gregarious forms suggests that the evolution of tolerant gregarious forms depends on much more stringent conditions. Thus, we should expect that the majority of independent evolutionary transitions from solitary to gregarious development should have occurred through the evolution of aggressive immobile larvae rather than the transition to tolerance.

\section{Sensitivity to Parameter Values}

To test the validity of our model, we altered values for the number of hosts in the habitat, the number of patches, the probability of patch location, and the ratio between the probability of patch location of solitary and gregarious parasitoids by $\pm 50 \%$ of their initial value. This analysis showed that our interpretations are robust. Model results changed quantitatively but not qualitatively.

\section{Discussion \\ Spatial Heterogeneity Facilitating the Evolution of Gregariousness}

The response of insect parasitoids to habitat spatial structure can be an important parameter in the evolution of the gregarious lifestyle of insect parasitoids. This is the first article that has explored how ecological factors like heterogeneity in host densities across patches and densitydependent behavioral responses of the parasitoids favor or disfavor the evolution of gregariousness. We see our ecological approach as additional to the population genetics approach used by previous authors.

The spread of gregarious development strongly depends on the probability of interaction between solitary and gregarious parasitoids. While previous work has concentrated on mechanisms that reduce this interaction within a single brood (Mayhew and Van Alphen 1999; Boivin and van Baaren 2000; Pexton and Mayhew 2001, 2002, 2004; Pexton et al. 2003), here we show that spatial processes across hosts provide opportunities for avoiding competition. First, the degree to which parasitoids exploit a given patch

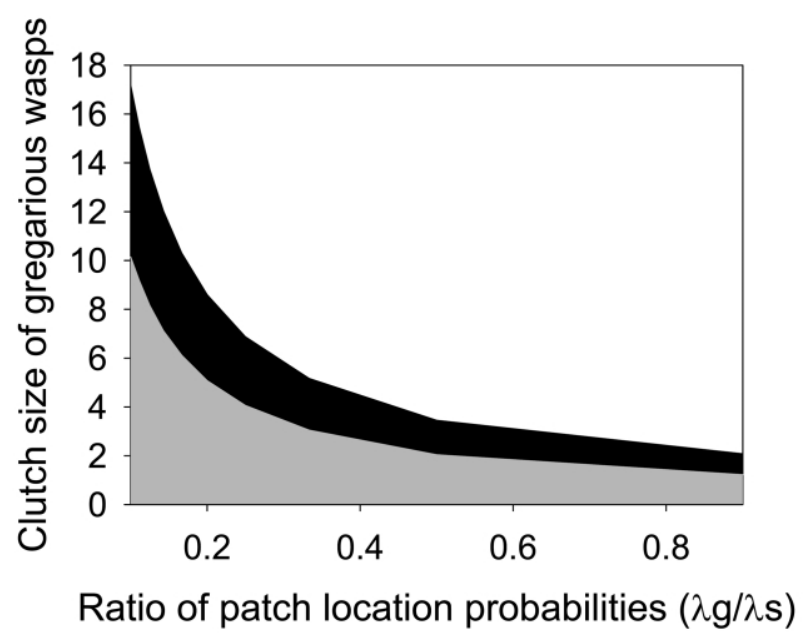

Figure 7: Parameter regions for the clutch size of gregarious wasps and the difference in patch location ability between solitary and gregarious wasps where a population of solitary wasps cannot be invaded by a gregarious mutant but a solitary mutant can invade a gregarious population (gray area), where a population of gregarious wasps can neither be invaded by a solitary mutant nor vice versa (black area), and where a population of gregarious wasps cannot be invaded by a solitary mutant but a gregarious mutant can invade a solitary population (white area). The ratio of patch location probability is expressed as the probability of a gregarious parasitoid to locate a given patch divided by the probability of a solitary parasitoid to locate a patch. Low $x$ values represent low patch location ability in gregarious parasitoids because of the life-history trade-off between longevity and fecundity; high $x$ values represent similar patch location ability in gregarious and solitary parasitoids. 
determines the overlap in host use between competitors. Optimal foraging theory predicts that patches are rarely fully exploited (Stephens and Krebs 1986), allowing gregarious mutants to have the sole use of individual hosts (scenario 1). Second, we show that host density-dependent use of infochemicals can lead to an aggregation of parasitoids in high-host-density patches, creating partial refuges in low-host-density patches for a gregarious mutant to avoid competition. Next, inverse density dependence in patch exploitation (scenario 4), as described for several host-parasitoid systems (Lessells 1985; Stiling 1987; Shiojiri and Takabayashi 2003), can also facilitate the evolution of gregariousness. This can, for example, be expected in host aggregations that aggressively defend themselves (Allen 1990).

\section{Spatial Heterogeneity Impeding the Evolution of Gregariousness}

When parasitoids enter and exploit patches in a host density-dependent fashion, solitary and gregarious parasitoid offspring are more likely to meet and compete. One mechanism that drives this host density-dependent parasitoid foraging comes from plants that release increasing amounts of volatiles as a function of increasing herbivore density (Vet and Dicke 1992; Geervliet et al. 1998).

\section{The Role of a Life-History Trade-Off}

If gregarious parasitoids invest more of their resources into fecundity, they should suffer a relatively short lifetime (e.g., Geervliet 1997; Pexton and Mayhew 2002). This may select for low dispersal, local search, and opportunism, that is, a generalist lifestyle, whereby parasitoids have an extended host range. Sheehan (1991) indeed found that gregariousness in parasitoids is significantly correlated with generalism. Gregariousness itself allows for the flexible allocation of variable clutches to differently sized hosts, an adaptive tool for a generalist. This egg allocation according to host size is impossible for the less flexible solitary parasitoids. Solitary parasitoids always produce only one offspring per host, even when they attack large hosts. Because there is an upper limit to their size on emergence, solitaries often leave large parts of an individual host unused (Harvey 1996; Harvey et al. 2000; Harvey and Strand 2002). Leaving host resources unused, which happens in all clades of the Ichneumonoid parasitoids that evolved gregarious forms, creates a huge opportunity for the evolution of gregariousness (J. Harvey, personal communication). Hence, in solitary hemolymph feeders, there is less selection for evolving a higher fecundity than for longevity and host specialization. We recognize this pattern in nature. The gregarious Cotesia glomerata lives shorter and attacks a much wider range of host species than the longer-lived solitary Cotesia rubecula, which is specialized on the widely dispersed small white butterfly Pieris rapae (Laing and Levin 1982; Nealis 1990; Geervliet 1997). This pattern may also apply to Aphaereta parasitoid species and Callosobruchus seed beetles (see Pexton and Mayhew 2002).

In this study, we did not address possible egg limitation in the parasitoids, an interesting issue in parasitoid foraging theory (Rosenheim 1996; Ellers et al. 2000a). We assumed sufficient plasticity in resource allocation being present (Ellers et al. 2000b). However, it is conceivable that at high host availability, especially gregarious mutants that lay multiple eggs per host deplete their egg supply and that this affects the spread of a mutant gene. This is certainly an issue that needs further elaboration.

\section{Large Host Aggregations}

Large host aggregations are likely to play a significant role in the evolution of gregariousness (scenario 3). They are also very interesting from a coevolutionary point of view. Herbivores can gain enemy-free space by assembling in a group (for selfish herd effects, see Hamilton 1971; Gross 1993). By grouping, herbivores, for example, can saturate the plant defense system and thus attract fewer natural enemies per capita. When these benefits are density dependent, selection for even larger aggregations may take place. In turn, once found, these very large aggregations select for high(er) fecundity in parasitoids, trading off with longevity. We expect that large host aggregations and their defense select for different responses in solitary and gregarious parasitoids. The reproductive output per host attacked is higher for gregarious parasitoids, and therefore being injured/killed by a defending host is a more severe penalty for a solitary parasitoid that needs to locate many hosts in its lifetime. As an example, the gregarious parasitoid species C. glomerata attacks the gregariously feeding and aggressively defending large white butterfly Pieris brassicae while the related solitary parasitoid species C. rubecula attacks the cryptic solitary P. rapae. Our results suggest that this is not a coincidence but a true result of spatial processes selecting for specific life-history traits. Data to test this hypothesis are extremely scarce. For this, we need a solid analysis of the spatial distribution of the hosts of congeneric pairs of solitary and gregarious parasitoids species.

An ecological approach toward understanding the evolution of gregariousness is challenging. The approach used here is not exhaustive with respect to influential parameters, and we did not aim for detailed realism. Future work could incorporate spatially explicit models, more realistic behavior, and an integration of ecological and population genetic approaches. 


\section{Acknowledgments}

A sabbatical visit of T.S.H. at the NIOO (KNAW) was supported by the Netherlands Organization for Scientific Research; L.E.M.V. spent a sabbatical leave at Simon Fraser University (Canada) supported by Wageningen University; B.D.R. was supported by a Natural Sciences and Engineering Research Council (Canada) operating grant. We acknowledge the critical comments of J. Burger, J. Harvey, L. Hemerik, K. Lessells, P. Mayhew, W. van der Putten, M. Visser, and two anonymous reviewers on earlier versions of the manuscript.

\section{APPENDIX}

\section{Models Linking Spatial Processes and Parasitoid Life History}

In our deterministic models, we focus on the probabilistic interactions of solitary and gregarious mutant offspring in hosts within a patch (when all patches are equally dense) or in a patch of each class of patch type (when classes of patches differ with regard to host densities). Parasitoids are distributed Poisson randomly across patches in the habitat. In scenarios where patches differ in their attractiveness to parasitoids, our parasitoid population is partitioned into subpopulations that reflect the differential attractiveness of patches based on their host density. Parasitoid distribution is Poisson random among patches of a given host-density class. Note that asymmetric interactions across patches that are colonized Poisson randomly differ greatly from interactions calculated from a patch with average parasitoid density, and thus all possible parasitoid densities per patch have to be computed. For the sake of simplicity, we assume that every parasitoid visits only a single patch (allowing for multiple patch visits would just increase the density of the interaction among parasitoids but would not change the pattern of interaction that we are interested in). Therefore, the probability of a patch being visited by a specific number of parasitoids $(p)$ can be well described by a Poisson distribution (fig. A1; see table A1 for symbols used).

We envision a habitat that contains $H$ hosts (fixed at 1,500) distributed among $N$ patches (fixed at 30) that either contain $h$ hosts each with $h=H / N$ or are subdivided into $k$ classes of patches with host densities that differ among but not within classes, with $\sum_{i=1}^{k} h_{i}=H k / N$; S solitary parasitoids (fixed at 99) plus a single mutant gregarious parasitoid search for these hosts. They locate patches with probability $\lambda_{s}$ and $\lambda_{g}$, respectively. Within patches, we assume random search for hosts and that parasitoids accept only unparasitized or conspecifically parasitized hosts; that is, no self-superparasitism occurs. We set our default value for the percentage of hosts that each parasitoid attacks within a patch $A$ to $A=0.6$ (for both solitary and gregarious parasitoids; table A1).

\section{The Spread of Tolerant (Nonfighting) Gregarious Mutant}

The expected reproductive success of a solitary parasitoid, our first measurement, depends only on the distribution of all 99 solitary parasitoids in the habitat. Note that the interaction with the tolerant gregarious parasitoid is irrelevant to the offspring of a solitary parasitoid and thus to the mother's reproductive success because gregarious larvae do not harm solitary larvae. Because the search for patches is random, the probability $\pi_{s}$ that a focal parasitoid finds itself in a patch with exactly $p_{s}$ solitary parasitoids is given by a Poisson distribution:

$$
\pi_{\mathrm{s}}\left(p_{\mathrm{s}}\right)=e^{\left(-\lambda_{s} s / N\right)} \frac{\left[\lambda_{\mathrm{s}}(S) / N\right]^{\left(p_{\mathrm{s}}\right)}}{\left(p_{\mathrm{s}}\right) !}
$$

with $p_{\mathrm{s}} \geq 1$. The reproductive success $F_{\mathrm{s}}\left(p_{\mathrm{s}}\right)$ that a solitary parasitoid with attack rate $A_{\mathrm{s}}$ achieves in a single visit to a patch with host density $h$ that it shares with $p_{\mathrm{s}}-1$ other solitary parasitoids is given by the fraction of hosts that were parasitized in this patch divided by the total number of solitary parasitoids exploiting the patch, $p_{\mathrm{s}}$ :

$$
F_{\mathrm{s}}\left(p_{\mathrm{s}}\right)=\frac{\left[1-\left(1-A_{\mathrm{s}}\right)^{p_{\mathrm{s}}}\right] h}{p_{\mathrm{s}}} .
$$

The value for the reproductive success of a solitary wasp $F_{s}$ is the sum of the probabilities of the occurrence of $p_{s}$ solitary wasps visiting the focal patch, which is given by $\pi_{\mathrm{s}}\left(p_{\mathrm{s}}\right)$, and the probability that our focal solitary parasitoid located the patch, $\lambda_{s}$. Thus, the total reproductive success a solitary parasitoid can expect in the focal patch is given by

$$
F_{s}=\lambda_{s} \sum_{p_{s}=1}^{S} \pi_{s}\left(p_{s}\right) F_{s}\left(p_{s}\right) .
$$

The chance that a gregarious mutant offspring finds itself without solitary competitors in a host, our second measurement, is determined by the proportion of gregarious offspring that experiences competition with solitary offspring. Given that all parasitoids that exploit the same patch comprise $p_{\mathrm{s}}$ solitary parasitoids and $p_{\mathrm{g}}$, the single mutant gregarious parasitoid, the gregarious parasitoid will encounter a fraction $A_{\mathrm{g}}$ of hosts in the patch, and among those, it will escape interaction with solitary offspring and develop uncontested only in the proportion $I_{\mathrm{g}}$ of hosts that was not attacked by other parasitoids. Thus, 


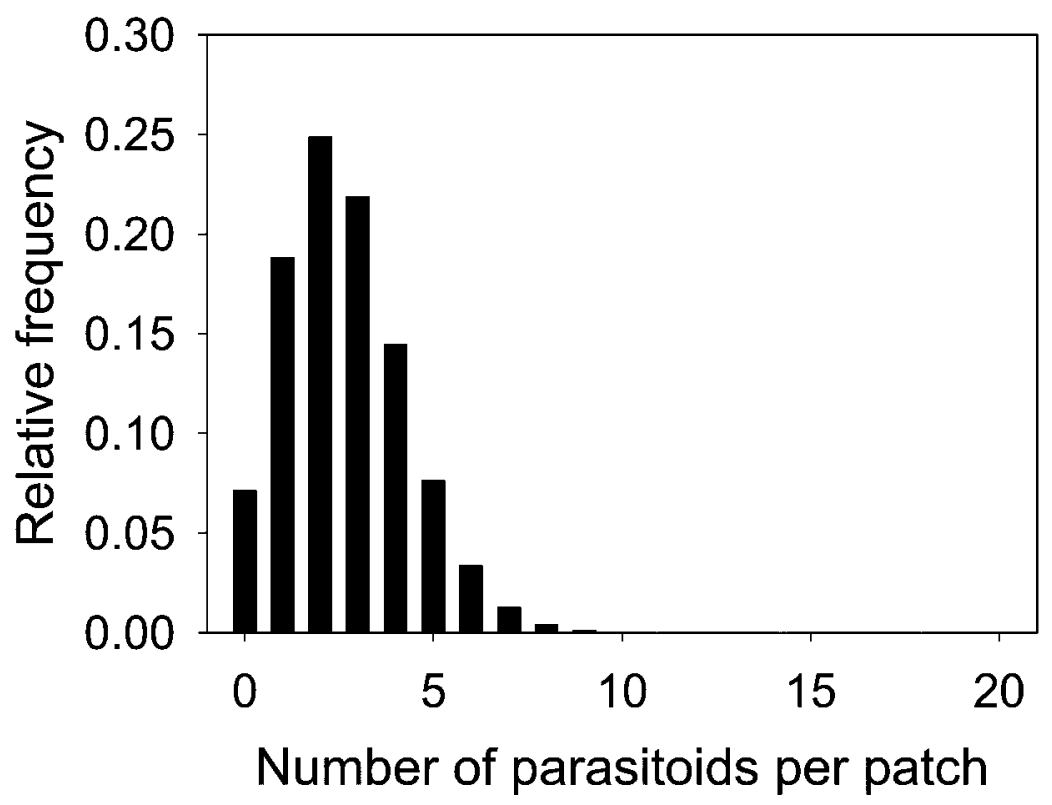

Figure A1: Frequency distribution of 99 solitary wasps that visit one of 30 patches Poisson randomly in the habitat.

$$
I_{\mathrm{g}}\left(p_{\mathrm{s}}\right)=\left(1-A_{\mathrm{s}}\right)^{p_{\mathrm{s}}} A_{\mathrm{g}}
$$

Multiplying $I_{\mathrm{g}}$ with the number of hosts that are in the focal patch gives the number $R_{\mathrm{g}}$ of hosts that was solely attacked by the gregarious parasitoid

$$
R_{\mathrm{g}}\left(p_{\mathrm{s}}\right)=\left[\left(1-A_{\mathrm{s}}\right)^{p_{\mathrm{s}}} A_{\mathrm{g}}\right] h
$$

The chance that the mutant will find herself in a patch with exactly $p$ parasitoids (i.e., $p_{\mathrm{s}}$ solitary and one gregarious parasitoid) is given by

$$
\pi_{\mathrm{g}}(p)=e^{\left[-\left(\lambda_{\mathrm{s}} S+\lambda_{\mathrm{g}}\right) / N\right]} \frac{\left[\left(\lambda_{\mathrm{s}} S+\lambda_{\mathrm{g}}\right) / N\right]^{(p)}}{(p) !}
$$

from a Poisson process that is based on the population of solitary parasitoids in the habitat, $S$, plus the gregarious mutant, that locate a patch with probability $\lambda_{s}$ in solitary and $\lambda_{\mathrm{g}}$ in mutant parasitoids. Given these probabilities, the probability that gregarious offspring in our focal patch develops in uncontested isolation is

$$
I_{\mathrm{g}}=\sum_{p=1}^{S+1} \pi_{\mathrm{g}}(p) I_{\mathrm{g}}\left(p_{\mathrm{s}}\right)
$$

Similarly, summing up across all probabilities of para- sitoid densities in a patch, the reproductive success of a gregarious mutant parasitoid is

$$
R_{\mathrm{g}}=\lambda_{\mathrm{g}} \sum_{p=1}^{S+1} \pi_{\mathrm{g}}(p) F_{\mathrm{g}}\left(p_{\mathrm{s}}\right) .
$$

A gregarious wasp needs to lay the marginal clutch size $\hat{C}$, our third measurement, into each host it attacks in order to achieve an equal reproductive success on a focal patch compared with a solitary parasitoid:

$$
\hat{C}=\frac{F_{\mathrm{s}}}{R_{\mathrm{g}}} .
$$

In other words, the reproductive success of our gregarious mutant, $F_{\mathrm{g}}$, is:

$$
F_{\mathrm{g}}=C R_{\mathrm{g}}
$$

in general and

$$
F_{\mathrm{g}}=\hat{C} R_{\mathrm{g}}
$$

when it lays the marginal clutch size. 
Table A1: Parameters used in the models for the appendix

\begin{tabular}{llr}
\hline Parameter & & Explanation \\
\hline$H$ & Number of hosts in the habitat & Default value \\
$h$ & Number of hosts in a patch & 1,500 \\
$N$ & Number of patches in the habitat & 50 \\
$k$ & Number of classes of patches each with a different host density & 30 \\
$S$ & Number of solitary parasitoids in the habitat & 10 \\
$p_{i}$ & Parasitoid density in patch $i$ & 99 \\
$A_{\mathrm{s}}$ & Proportion of hosts a solitary parasitoid parasitizes in a patch & .6 \\
$A_{\mathrm{g}}$ & Proportion of hosts a gregarious parasitoid parasitizes in a patch & .6 \\
$\lambda_{\mathrm{s}}$ & Probability of a solitary parasitoid to enter a patch & .8 \\
$\lambda_{\mathrm{g}}$ & Probability of a gregarious parasitoid to enter a patch & .8 \\
$\pi_{\mathrm{s}}(p)$ & Probability of a solitary parasitoid to be in a patch with parasitoid density $p$ & \\
$\pi_{\mathrm{g}}(p)$ & Probability of a gregarious parasitoid to be in a patch with parasitoid density $p$ & \\
$f$ & Proportion of the parasitoid population that forages within a patch class of a given host density & \\
\hline
\end{tabular}

\section{Scenarios with Spatial Heterogeneity in Host Density across Patches}

In scenarios 2-4, we divide the habitat into $k=10$ classes of patches with host densities that vary between five and 95 hosts per patch, that is, for $k \in$ $\{1,2, \ldots, 9,10\} h(k)=10 k-5$. In the most simple case, we assume that the probability of patch location $\left(\lambda_{\iota}\right)$ is independent of $h(k)$. Our three measurements, $F_{s}, I_{\mathrm{g}}$, and $\hat{C}$, are calculated separately for each host density class.

The reproductive success of the solitary parasitoid in patch class $k, R_{s}(k)$, the probability of exploiting a hosts uncontested, $I(k)$, and the marginal clutch size in that patch, $\hat{C}(k)$, are calculated in the same way as in the previous scenario. These values must be weighted by the proportion of parasitoids $f$ that forages within each patch class to obtain the average values of our three measurements $\overline{R_{s}}, \bar{I}$, and $\bar{C}$ :

$$
\begin{aligned}
& \overline{F_{\mathrm{s}}}=\sum_{k=1}^{10} \frac{f F_{\mathrm{s}}(k)}{10}, \\
& \overline{I_{\mathrm{g}}}=\sum_{k=1}^{10} \frac{f I_{\mathrm{g}}(k)}{10}, \\
& \bar{C}=\sum_{k=1}^{10} \frac{f \hat{C}(k)}{10} .
\end{aligned}
$$

Note that while the proportion of the parasitoid population that enters a patch of a given class of patches can vary in this scenario, the attack rate of solitary and gregarious parasitoids within patches remains constant $(A=0.6)$ across all host density classes.

\section{Numerical Solution for the Invasion of a Population of Solitary Parasitoids by a Gregarious Mutant with Aggressive Larvae}

Conditions for the invasion of a population of solitary parasitoids by a gregarious mutant parasitoid were solved as an individual-based stochastic model based on scenario 4. A Poisson distribution of parasitoids across patches was realized by randomly assigning each of the $S=99$ solitary parasitoids with probability $\lambda_{\mathrm{s}}=0.8$ to one of $N=30$ patches that contained $H=50$ hosts each. Random host search with attack rate $A$ was realized by letting each solitary parasitoid that visited a patch lay a single egg into each host of the patch with probability $A=0.6$. In a similar way, the gregarious mutant was assigned to one of the patches with probability $\lambda_{\mathrm{g}}$ (with $\lambda_{\mathrm{g}}$ varied as described in scenario 4) and allowed to oviposit into hosts with attack rate $A$, except that the gregarious mutant could lay a clutch of size $C$. In hosts superparasitized by solitary parasitoids only, a single offspring survived per host. In hosts multiparasitized by solitary parasitoids and the gregarious mutant, survival was determined by Monte Carlo simulation. Given that at least one solitary and one gregarious larva were present in a host, one of the solitary larvae was drawn randomly, and from the remaining larvae, an opponent was randomly drawn. (Note that only solitary larvae were drawn as first larva because only solitary mobile larvae can encounter other larvae.) A random draw with $p=$ 0.5 decided who of the opponents would survive. This was iterated until either only solitary larvae remained (in which case the above mentioned condition applied) or only gregarious larvae remained. If more than one gregarious parasitoid larva survived, it was assumed that all could develop into mature insects. Because the outcome of interactions between solitary and gregarious larvae is not a linear function of the clutch size $C$ of gregarious wasps, 
$\hat{C}$ could not be calculated as described above. Simulations were started with $C=1$, and $C$ was subsequently increased until, for a given value of the probability of patch location in gregarious wasps $\lambda_{\mathrm{g}}$ gregarious and solitary wasps achieved equal reproductive success.

\section{Numerical Solution for the Invasion of a Population of Gregarious Parasitoids with Tolerant Larvae by a Solitary Mutant}

The invasion of a solitary mutant into a gregarious wasp population was solved numerically in a stochastic simulation because the analytical solution of all possible densities of gregarious larvae in a given host was computationally too cumbersome. Again, the simulation was based on scenario 4, with the probability of patch location by gregarious wasps being varied. For each individual of a population of 99 gregarious wasps, it was determined by $\lambda_{\mathrm{g}}$ whether or not it found a patch, and given success, wasps were Poisson randomly distributed across 30 patches containing 50 hosts each. As described above, each female was allowed to attack hosts in a patch with $A=0.6$ probability flip of a biased coin. The single mutant solitary wasp found a patch with $\lambda_{s}$ and was randomly assigned to one of the 30 patches. The mutant attacked each host with $A=0.6$ probability. Because the gregarious population was considered to represent tolerant individuals, all gregarious offspring in hosts that were also attacked by a solitary larva were killed in competition. The reproductive success of the solitary mutant was solely based on the number of hosts she could attack. The reproductive success of gregarious wasps depended on the number of gregarious offspring that were able to develop in hosts not attacked by the solitary mutant, divided by the number of gregarious adults in the population. The marginal clutch size $\hat{C}$ was determined by dividing the reproductive success of the solitary mutant by the reproductive success of a gregarious female with clutch size $C=1$.

\section{Literature Cited}

Allen, G. R. 1990. Influence of host behavior and host size on the success of oviposition of Cotesia urabae and Dolichogenidea eucalypti (Hymenoptera: Braconidae). Journal of Insect Behavior 3: 733-749.

Bertram, B. C. R. 1980. Vigilance and group size in ostriches. Animal Behaviour 28:278-286.

Boivin, G., and J. van Baaren. 2000. The role of larval aggression and mobility in the transition between solitary and gregarious development in parasitoid wasps. Ecology Letters 3:469-474.

Dicke, M., and M. W. Sabelis. 1988. Infochemical terminology: based on a cost benefit analysis rather than origin of compounds. Functional Ecology 2:131-139.

Ellers, J., J. G. Sevenster, and G. Driessen. 2000a. Egg load evolution in parasitoids. American Naturalist 156:650-665.
Ellers, J., G. Driessen, and J. G. Sevenster. 2000b. The shape of the trade-off function between egg production and life span in the parasitoid Asobara tabida. Netherlands Journal of Zoology 50:2936.

Geervliet, J. B. F. 1997. Infochemical use by insect parasitoids in a tritrophic context: comparison of a generalist and a specialist. $\mathrm{PhD}$ thesis. Wageningen University.

Geervliet, J. B. F., S. Ariens, M. Dicke, and L. E. M. Vet. 1998. Longdistance assessment of patch profitability through volatile infochemicals by the parasitoids Cotesia glomerata and C. rubecula (Hymenoptera: Braconidae). Biological Control 11:113-121.

Godfray, H. C. J. 1987. The evolution of clutch size in parasitic wasps. American Naturalist 129:221-233.

. 1994. Parasitoids: behavioral and evolutionary ecology. Princeton University Press, Princeton, NJ.

Gross, P. 1993. Insect behavioral and morphological defenses against parasitoids. Annual Review of Entomology 38:251-273.

Hamilton, W. D. 1971. Geometry for the selfish herd. Journal of Theoretical Biology 31:295-311.

Harvey, J. A. 1996. Venturia canescens parasitizing Galleria mellonella and Anagasta kuehniella: is the parasitoid a conformer or regulator? Journal of Insect Physiology 42:1017-1025.

Harvey, J. A., and M. R. Strand. 2002. The developmental strategies of endoparasitoid wasps vary with host feeding ecology. Ecology 83:2439-2451.

Harvey, J. A., K. Kadash, and M. R. Strand. 2000. Differences in larval feeding behavior correlate with altered developmental strategies in two parasitic wasps: implications for the size-fitness hypothesis. Oikos 88:621-629.

Harvey, P. H., and L. Partridge. 1987. Murderous mandibles and black holes in hymenopteran wasps. Nature 326:128-129.

Krebs, J. R., and N. B. Davies. 1993. An introduction to behavioural ecology. Blackwell Scientific, Oxford.

Laing, J. E., and D. B. Levin. 1982. A review of the biology and a bibliography of Apanteles glomeratus (L.) (Hymenoptera: Braconidae). Biocontrol News and Information 3:7-23.

Lessells, C. M. 1985. Parasitoid foraging: should parasitism be density dependent? Journal of Animal Ecology 54:27-41.

Mayhew, P. J. 1998a. The evolution of gregariousness in parasitoid wasps. Proceedings of the Royal Society of London B 265:383389.

- 1998b. The life-histories of parasitoid wasps developing in small gregarious broods. Netherlands Journal of Zoology 48:225240.

Mayhew, P. J., and I. C. W. Hardy. 1998. Nonsiblicidal behavior and the evolution of clutch size in bethylid wasps. American Naturalist 151:409-424.

Mayhew, P. J., and J. J. M. Van Alphen. 1999. Gregarious development in alysiine parasitoids evolved through a reduction in larval aggression. Animal Behaviour 58:131-141.

Mayhew, P. J., P. J. Ode, I. C. W. Hardy, and J. A. Rosenheim. 1998. Parasitoid clutch size and irreversible evolution. Ecology Letters 1:139-141.

Nealis, V. G. 1990. Factors affecting the rate of attack by Cotesia rubecula (Hymenoptera: Braconidae). Ecological Entomology 15: 163-168.

Neill, S. R. S. J., and J. M. Cullen. 1974. Experiments on whether schooling by their prey affects the hunting behaviour of cephalopods and fish predators. Journal of Zoology (London) 172:549569. 
Ode, P. J., and J. A. Rosenheim. 1998. Sex allocation and the evolutionary transition between solitary and gregarious parasitoid development. American Naturalist 152:757-761.

Perez-Contreras, T., J. J. Soler, and M. Soler. 2003. Why do pine processionary caterpillars Thaumetopoea pityocampa (Lepidoptera, Thaumetopoeidae) live in large groups? an experimental study. Annales Zoologici Fennici 40:505-515.

Pexton, J. J., and P. J. Mayhew. 2001. Immobility: the key to family harmony? Trends in Ecology \& Evolution 16:7-9.

2002. Siblicide and life-history evolution in parasitoids. Behavioral Ecology 13:690-695.

- 2004. Competitive interactions between parasitoid larvae and the evolution of gregarious development. Oecologia (Berlin) 141:179-190.

Pexton, J. J., D. J. Rankin, C. Dytham, and P. J. Mayhew. 2003. Asymmetric larval mobility and the evolutionary transition from siblicide to nonsiblicidal behavior in parasitoid wasps. Behavioral Ecology 14:182-193.

Prokopy, R. J., and B. D. Roitberg. 2001. Joining and avoidance behavior in nonsocial insects. Annual Review of Entomology 46: 631-665.

Roff, D. A. 1992. The evolution of life histories. Chapman \& Hall, New York.

Rohlfs, M., and T. S. Hoffmeister. 2003. An evolutionary explanation of the aggregation model of species coexistence. Proceedings of the Royal Society of London B 270(suppl.):33-35.

Rosenheim, J. A. 1993. Single-sex broods and the evolution of nonsiblicidal parasitoid wasps. American Naturalist 141:90-104.

. 1996. An evolutionary argument for egg limitation. Evolution 50:2089-2094.

Rosenheim, J. A., and D. Hongkham. 1996. Clutch size in an obligately siblicidal parasitoid wasp. Animal Behaviour 51:841-852.

Sevenster, J. G., J. Ellers, and G. Driessen. 1998. An evolutionary argument for time limitation. Evolution 52:1241-1244.

Sheehan, W. 1991. Host range patterns of hymenopteran parasitoids of exophytic lepidopteran folivores. Pages 209-248 in E. Bernays, ed. Insect-plant interactions. CRC, Boca Raton, FL.

Shiojiri, K., and J. Takabayashi. 2003. Effects of specialist parasitoids on oviposition preference of phytophagous insects: encounterdilution effects in a tritrophic interaction. Ecological Entomology 28:573-578.

Stearns, S. C. 1992. The evolution of life histories. Oxford University Press, Oxford.

Stephens, D. W., and J. R. Krebs. 1986. Foraging theory. Princeton University Press, Princeton, NJ.

Stiling, P. D. 1987. The frequency of density dependence in insect host-parasitoid systems. Ecology 68:844-856.

van Alphen, J. J. M., and M. E. Visser. 1990. Superparasitism as an adaptive strategy for insect parasitoids. Annual Review of Entomology 35:59-79.

van Dijken, M. J., P. van Stratum, and J. J. M. van Alphen. 1992. Recognition of individual-specific marked parasitized hosts by the solitary parasitoid Epidinocarsis lopezi. Behavioral Ecology and Sociobiology 30:77-82.

Vet, L. E. M., and M. Dicke. 1992. Ecology of infochemical use by natural enemies in a tritrophic context. Annual Review of Entomology 37:141-172.

Vinson, S. B. 1984. How parasitoids locate their hosts: a case of insect espionage. Pages 325-348 in T. Lewis, ed. Insect communication. Academic Press, London.

Visser, M. E. 1993. Adaptive self- and conspecific superparasitism in the solitary parasitoid Leptopilina heterotoma (Hymenoptera: Eucoilidae). Behavioral Ecology 4:22-28.

Wertheim, B., E.-J. A. van Baalen, M. Dicke, and L. E. M. Vet. 2005. Pheromone-mediated aggregation in nonsocial arthropods: an evolutionary ecological perspective. Annual Review of Entomology 50:321-346.
Associate Editor: Peter D. Taylor Editor: Michael C. Whitlock 